\section{Improved power-law- detector-based moving small dim target detection in infrared images}

Bin Wu and Hong-Bing Ji

Xidian University, School of Electronic Engineering, P.O.Box 133, Xidian University, Xi'an 710071, China

E-mail: wubin_326@ hotmail.com

\begin{abstract}
A novel algorithm based on an improved powerlaw detector is proposed to detect moving small dim targets against heavy cluttered background in IR image sequences. As the gray-scale value of a pixel in an image fluctuates when a target passes by, this fluctuation can be viewed as a weak transient signal, and can be detected by the power-law detector, which performs well for transient signal detection. The experimental results illustrate that the proposed algorithm is effective and adaptable for moving small dim target detection in image sequences with heavy clutter. @ 2008 Society of Photo-Optical Instrumentation Engineers.

[DOI: $10.1117 / 1.2829771]$
\end{abstract}

Subject terms: target detection; transient signal; power-law detector; infrared image sequence.

Paper 070644LR received Aug. 1, 2007; revised manuscript received Oct. 19, 2007; accepted for publication Oct. 29, 2007; published online Jan. 8, 2008.

\section{Introduction}

Recently, the detection of moving small dim targets under heavy IR background clutter has been an active research area, and many algorithms for this problem have been developed. ${ }^{1-4}$ The algorithms used in IR searching and tracking systems are adequate for applications with bright targets against background clutter, and make use of only the spatial information of the targets and clutter without considering their temporal behavior. Some new approaches ${ }^{2,3}$ incorporated temporal and spatial information have good performance for the detection of small dim targets in IR image sequences, but heavy computational complexity is a frustration in practical applications.

Since that the temporal profile of a pixel through which a target passes is different from those through which clutter pass, Tzannes and Brooks ${ }^{3}$ developed the models of temporal behavior of clutter and targets on a single-pixel basis. These are used to develop a temporal likelihood-ratio test for target detection. Being different from the mentioned algorithm based on 1-D signal procession, our algorithm views the fluctuation of the gray-scale value of a pixel, while a target passes, as a weak transient signal. Owing to the good performance for transient signal detection in Gaussian noise, the power-law detector ${ }^{5,6}$ is employed in our algorithm to detect small dim targets.

The rest of the paper is organized as follows. Section 2 describes the power-law detector and the motivation behind the use of the power-law detector for small dim target detection in an IR image. Section 3 describes our algorithm.

0091-3286/2008/\$25.00 @ 2008 SPIE

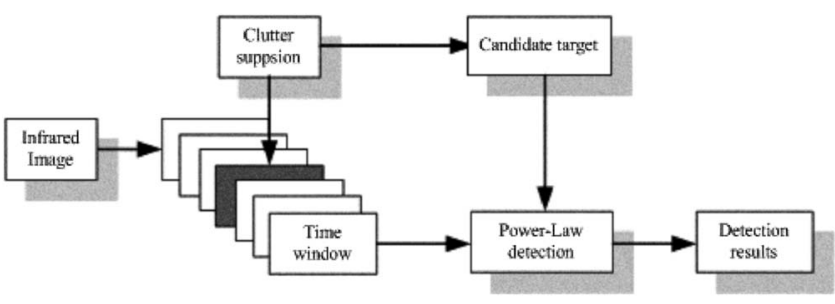

Fig. 1 Flowchart of the algorithm

Section 4 presents experimental results with corresponding analyses. Finally, conclusions are given in Sec. 5.

\section{Power-Law Detector}

The power-law detector, developed by Nuttall, ${ }^{5}$ performs well for transient signal detection without knowledge of the signal, hence its wide applications. In a Gaussian background, Nuttall considered that the transient signal detection can be transformed into the problem of arbitrary $M$ bits signal detection among the $N$ discrete Fourier transform (DFT) bins, where $M$ is the number of signal-present bins. For a 1-D signal $x(t)$ including a transient signal, the power-law statistic can be formulated as

$\sum_{j=1}^{N}|X(j)|^{v} \begin{cases}\leqslant \lambda & \text { signal absent } \\ >\lambda & \text { signal present, }\end{cases}$

where $X(j)$ is the $j$ th magnitude-squared DFT bins, $N$ is the total number of DFT bins, $\lambda$ the threshold, and $v$ is a positive real number with an empirical value of 2.5 .

Given the contiguity tendency of a transient signal in frequency, Willett and Wang ${ }^{6}$ modified $X(j)$ in Eq. (1) and obtained new random variables, i.e., $W_{j}=X_{j-1}+X_{j}, j$ $=1, \ldots, N$ by combining two contiguous frequency bins. A new power-law detector is defined as

$T(W)=\sum_{j=1}^{N} W_{j}^{v}=\sum_{j=1}^{N}\left(X_{j-1}+X_{j}\right)^{v} \begin{cases}\leqslant \lambda & \text { signal absent } \\ >\lambda & \text { signal present. }\end{cases}$

The power-law detector and its improved version are nonparametric detectors, and prior knowledge of the transient signal such as the model or the frequency spectrum is not necessary. According to the assumption that the temporal behavior of the background noise is additive Gaussian noise ${ }^{3}$ the fluctuation of the gray-scale value of a pixel in temporal profiles caused by a passing target can be viewed as a transient signal, and its detection is transformed into the problem of transient signal detection in Gaussian noise.

\section{Algorithm Implementation}

The algorithm consists of two parts, clutter suppression in the spatial domain and power-law detection in the time domain, as shown in Fig. 1.

First, a time window with length of $L$ frames is introduced. The temporal profile of each pixel can be viewed as a 1-D signal in the time window. To decrease the computational burden, the current image can be processed by a max-median filter ${ }^{7}$ in the spatial domain to suppress the 


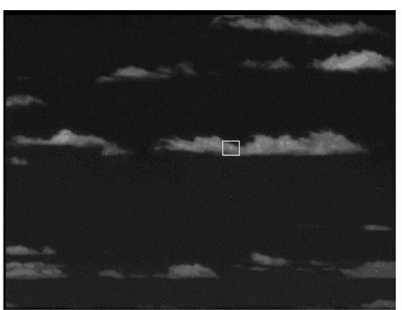

(a)

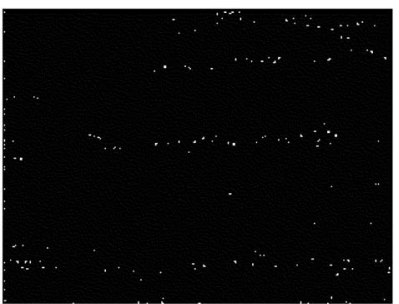

(c) (b)

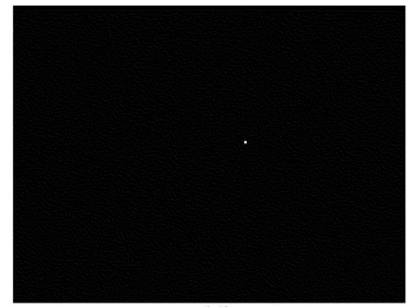

(d)

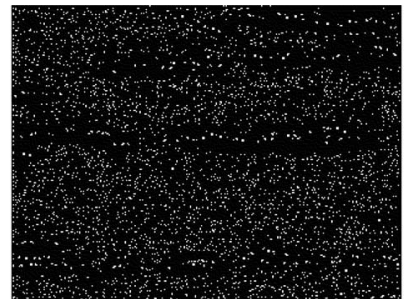

Fig. 2 Simulation results of IR image sequence: (a) the fifteenth frame of the IR image sequence, (b) the image after max-median filtering, (c) the image after self-adapted valve segmentation, and (d) the detection result.

clutter and obtain the candidate target pixels. Then the power-law detector is used to detect real target on the temporal profiles of the candidate target pixels. Nevertheless, to let the "pulse" fully present in the time window, the current image is put at the center of the time window. Then, the length of the window is related to the size and the speed of the transient signal. ${ }^{3}$ Generally, $L$ is set as 7 to 15 .

With the candidate pixels by the max-median filter ${ }^{7}$ just obtained, the small dim targets detection problem can be formulated as a binary hypothesis test:

$H_{0}: \quad F(x, y, n)=N(x, y, n)$,

$H_{1}: \quad F(x, y, n)=T(x, y, n)+N(x, y, n)$,

where $F(x, y, n)$ is the gray-scale value of the candidate pixel at position $(x, y)$ at time $n ; T(x, y, n)$ is the gray-scale value of a target that is a transient signal; $N(x, y, n)$ is the Gaussian noise independent of the $T(x, y, n) ; n$ is time index; and $n=1,2, \ldots, N$. Otherwise, due to the contiguity of a transient signal in frequency domain, the modified powerlaw statistic in Eq. (2) is adopted to improve the detection performance. Thus, the binary hypothesis test is transformed into the problem of transient signal detection in an IR image

$T(W)=\sum_{j=1}^{N} W_{j}^{v}=\sum_{j=1}^{N}\left(X_{j-1}+X_{j}\right)^{v} \begin{cases}\leqslant \lambda & H_{0} \\ >\lambda & H_{1},\end{cases}$

where $X(j)$ is the $j$ 'th magnitude-squared DFT bin, $\lambda$ is the threshold, and $v$ is a positive real number.

Similar to the description in the work of Wang and Willet, ${ }^{6} T(W)$ is also rewritten as

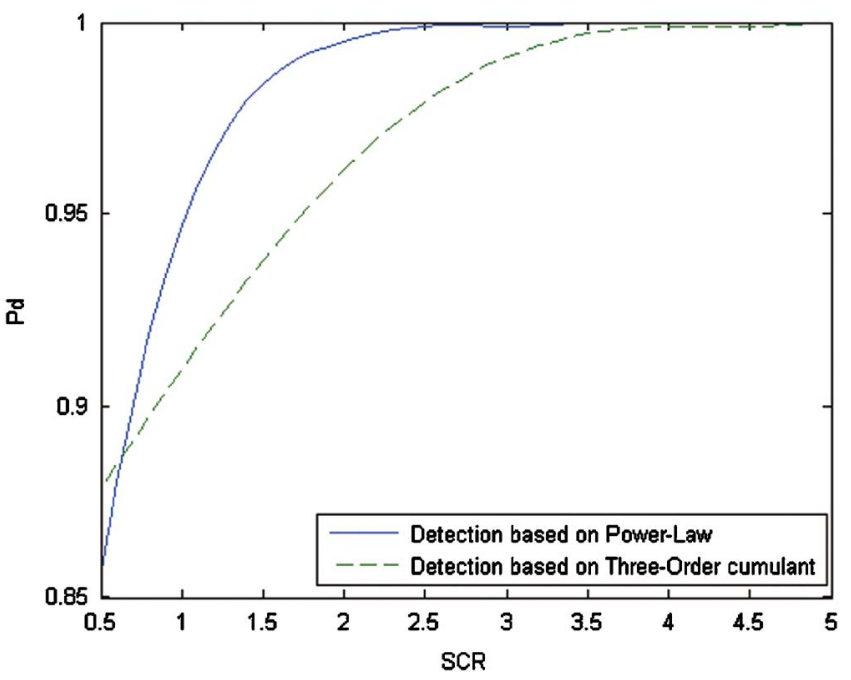

Fig. 3 Detection performance versus SCRs of our algorithm and the third-order-cumulant-based algorithm $\left(P_{\mathrm{FA}}=10^{-4}\right)$.

$$
\begin{aligned}
T(W)= & \sum_{j=1}^{N} W_{j}^{v}=\sum_{j=1}^{N}\left(X_{j-1}+X_{j}\right)^{v}=\sum_{j=1}^{N / 2} W_{2 j-1}+\sum_{j=1}^{N / 2} W_{2 j}=T_{o} \\
& +T_{e} .
\end{aligned}
$$

As analysed in the literature, ${ }^{6} X_{j}$ follows an independent and identically distributed (i.i.d) exponential distribution under hypothesis $H_{0}$, and $F(x, y, n)$ is approximately Gaussian noise. Because that $W_{2 j-1}$ and $W_{2 j}, j$ $=1, \ldots, N / 2$, are i.i.d exponential variables, $T_{o}$ and $T_{e}$ converge to the distribution $N\left(\mu, \sigma^{2}\right)$ via the central limit theorem, with

$\mu=\frac{N}{2} \Gamma(v+2)$,

$\sigma^{2}=\frac{N}{2}\left[\Gamma(2 v+2)-\Gamma(v+2)^{2}\right]$.

Due to $T(W)=T_{0}+T_{e}, \quad T(W)$ follows the distribution $N\left(2 \mu, 4 \sigma^{2}\right)$ when the power law $v=2.5$. A detailed derivation is found in Ref. 6 . Therefore, given the probability of false alarms $P_{\mathrm{FA}}$, the threshold $\lambda$ can be computed from the normal distribution.

\section{Experiments and Analysis}

The signal-to-clutter ratio (SCR) is defined as

$\operatorname{SCR}=\frac{a^{2}}{\sigma^{2}}$

where $a^{2}$ denotes the target intensity, and $\sigma^{2}$ is the variance of background clutter.

\subsection{Experiment 1}

A target of size $2 \times 2$ is embedded in an IR image sequence of 95 frames, which goes through the cloud along a straight line. The parameters of the algorithm are selected as follows: the false alarm probability $P_{\mathrm{FA}}=10^{-4}$, thus the result- 


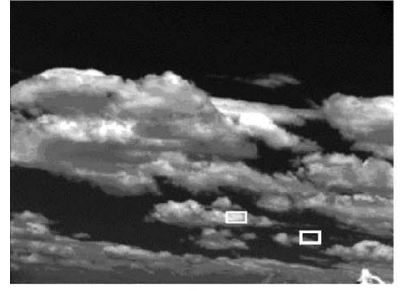

(a)

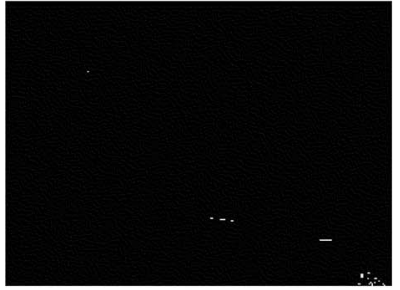

(b)

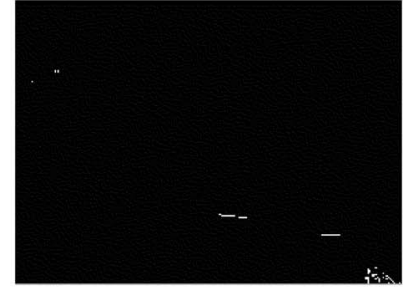

(c)

Fig. 4 Performance comparison of the Tzannes and Brooks algorithm and our algorithm: (a) single frame of the sequence, (b) the results of the Tzannes and Brooks algorithm, and (c) the results of our algorithm.

ing threshold $\lambda=3200$, the length of sliding window $L=9$, and the length of the DFT $N=128$. Note that the effective detection begins from the fifth frame. Figure 2 shows the detection results with $\mathrm{SCR}=1$ for our algorithm. Figure 3 illustrates the detection performances with different SCRs of our algorithm and the third-order-cumulant ${ }^{4}$ based algorithm.

When $\mathrm{SCR}=1.0$, only a few of possible target pixels are detected after max-median filtering and self-adapted valve segmentation, ${ }^{7}$ as shown in Fig. 2(c). Then, for these pixels, our algorithm is used for further detection and the results are shown in Fig. 2(d). Note that our algorithm can detect the small gray change caused by a target passing through the clouds. From Fig. 3, it is clear that our algorithm has a better performance than the third-order-cumulant-based algorithm for $\mathrm{SCR} \geq 0.7$. However, due to the limit of clutter suppression, the detection probabilities decline sharply when SCR $<0.7$, while the third-order-cumulant-based algorithm also has good performance.

\subsection{Experiment 2}

The second sequence is acquired by a PtSi IR cameras with focal plane arrays ${ }^{3}$ of $320 \times 244$ pixels. The selected sequence of 95 consecutive frames is used for the algorithm evaluation. Figure 4(a) shows a single image of this sequence, which is a daytime scene that includes two point targets and many drifting and evolving clouds. The left target is detected accurately by our algorithm in 85 frames, while the Tzannes and Brooks algorithm detects the target in just 68 frames, as shown in Figs. 4(b) and 4(c). Due to the higher SCR of the right target, there is little difference of detection performance between the two algorithms.

\section{Conclusions}

We presented a novel algorithm based on the improved power-law detector for the detection of moving small dim targets in image sequences with heavy clutter. It was heuristically demonstrated that the proposed algorithm is very successful for the detection of pixel-sized target. The effectiveness of the algorithm was demonstrated with two experiments. The experimental results illustrate that our algorithm can promote the performance of small dim target detection under heavy clutter background.

\section{Acknowledgments}

This work is supported by the National Natural Science Foundation of China (No. 60677040).

\section{References}

1. S. D. Blostein and T. S. Huang, "Detection of small moving objects in image sequences using multistage hypothesis testing," IEEE Trans. Signal Process. 39(7), 1611-1629 (1991).

2. S. S. Blackman, "Multiple hypothesis tracking for multiple target tracking," IEEE Aerosp. Electron. Syst. Mag. 19(1), 5-18 (2004).

3. A. P. Tzannes and D. H. Brooks, "Detection small moving objects using temporal hypothesis testing," IEEE Trans. Aerosp. Electron. Syst. 38(2), 570-585 (2002).

4. WU Bin, JI Hongbing, and LI Peng, "A new method for moving dim target detection based on third-order cumulant in Infrared image," Int. J. Infrared Millim. Waves 25(5), 364-367 (2006).

5. A. Nuttall, "Detection performance of power-law processors for random signals of unknown location, structure, extent, and strength," NUWC-NPT Technical Report, 10 751, Newport, RI (Sep. 1994).

6. Z. Wang and P. Willett, "All-purpose and plug-in power-law detectors for transient signals," IEEE Trans. Signal Process. 49(11), 24542466 (2001).

7. S. D. Deshpande, M. H. Er, V. Ronda, and P. Chan, "Max-mean and max-median filters for detection of small targets," in Signal and Data Processing of Small Targets, Proc. SPIE 3809, 74-83 (1999). 